\title{
Bias Adaptive Statistical Inference Learning Agents for Learning from Human Feedback
}

\author{
Jonathan Watson \\ University of Kentucky \\ jonathan. wat son@uky . edu
}

\author{
Brent Harrison \\ University of Kentucky \\ harrisonecs.uky. edu
}

\begin{abstract}
We present a novel technique for learning behaviors from a human provided feedback signal that is distorted by systematic bias. Our technique, which we refer to as BASIL, models the feedback signal as being separable into a heuristic evaluation of the utility of an action and a bias value that is drawn from a parametric distribution probabilistically, where the distribution is defined by unknown parameters. We present the general form of the technique as well as a specific algorithm for integrating the technique with the TAMER algorithm for bias values drawn from a normal distribution. We test our algorithm against standard TAMER in the domain of Tetris using a synthetic oracle that provides feedback under varying levels of distortion. We find our algorithm can learn very quickly under bias distortions that entirely stymie the learning of classic TAMER.
\end{abstract}

\section{Introduction}

Today, machine learning plays an ever-increasing role in the development of autonomous agents. One of the major approaches to machine learning is reinforcement learning (Sutton, Barto, and others 1998) where the agent learns behavior from a reward signal. In many traditional approaches to reinforcement learning, the reward signal comes from the agent's environment, possibly interpreted by a heuristic function. However, the reward signal for an agent can be delayed several actions or time-steps from the action that ultimately lead to the reward, resulting in the agent struggling to associate the desired behavior with the reward. Interactive reinforcement learning allows a human trainer to provide a reward signal either in addition to or in replacement of an environmentally produced signal and human trainers are often able to recognize that an action will have long term benefits and provide an more immediate reward signal that an agent can more easily associate with the relevant action. Knox and Stone showed that even without access to an environmental reward signal, an agent learning from a human trainer could very quickly reach a high level of performance with a small number of training episodes (Knox and Stone 2009).

The introduction of a human trainer is not without drawbacks. Humans have limited time to devote to training tasks and the rate at which they can provide feedback is also limited. Of more concern, introducing a human trainer adds hu-

Copyright (C 2021 by the authors. All rights reserved. man factors to the feedback signal that are not universally well understood. Work by Bartneck, Reichenbach, and Carpenter (Bartneck, Reichenbach, and Carpenter 2008) found that aspects of an agent's presentation affected feedback given by human trainers. We will discuss this work further in the related work section as it is one that motivates our contribution. Work by Loftin et.al. (Loftin et al. 2014) found that aspects of the trainers had a significant effect on the feedback they gave. Their work also provided two algorithms to improve learning from human trainers giving inconsistent feedback. However, the algorithms they provide assume that feedback is given only in a discrete form of positive, negative, or zero; and that for any given state human trainers should consider only one on policy action as worthy of receiving reward. We will also discuss this work further in later sections.

We argue that when provided the means to communicate more detailed numerical rewards, human trainers can provide an agent with a heuristic estimate of the utility of taking a given action in a given state. We model numerical human feedback as being separable into an assessment of the utility of taking a given action in a given state and a biasing value, which is drawn from a probabilistic distribution. Handling this bias is important because, as our experiments show, it can have a negative effect on learning and in more extreme cases prevent it entirely in existing techniques. Bias is also an inherent risk of using human feedback. As far as we know, no existing technique handles this sort of bias in numerically valued rewards to interactive reinforcement learning agents. Existing techniques for such agents are limited to handling human factors in discrete feedback and do not handle general forms of bias, but are focused on other human factors such as signal withholding, which limits the sorts of problems they are suited for.

We present a general technique for separating the utility evaluation and biasing values from a history of reward signals when given the form of the biasing distribution, but without knowing the parameters of the distribution using expectation maximization (EM) (Dempster, Laird, and Rubin 1977) to compute a maximum likelihood estimate of the utility assessments. Additionally, we provide an algorithm utilizing this technique for a normal distribution and test it against several other algorithms under a variety of biasing effects and permutations of parameters using simulated hu- 
man trainers.

\section{Related Work}

The recent past has seen a growing body of work in the area of agents learning from human feedback. Thomaz and Breazeal (Thomaz and Breazeal 2006) conducted an experiment where human trainers helped a virtual agent to learn to perform a simulated cake baking task. Among other findings, they observed that human trainers gave rewards in anticipation of good actions, not just for immediate positive outcomes, and that the feedback from human trainers corresponds more to an action's utility than to the immediate reward it provided. Knox and Stone (Knox and Stone 2009) provided the TAMER algorithm which they showed learned very quickly to perform moderately well in the complicated task of the game Tetris.

There has been some work on how human factors influence feedback given by a human trainer such as experiments performed by Bartneck, Reichenbach, and Carpenter (Bartneck, Reichenbach, and Carpenter 2008) where they found that aspects of an agent's presentation affected feedback given by human trainers even though the agents used identical algorithms, and had no differences beyond visual representation and name. They had their agents represented by robots with differing degrees of anthropomorphism. They found significant differences in how human trainers provided feedback between each of the representation.

Research by Knox et.al. (Knox et al. 2012) investigated differences in feedback provided by human trainers when those human trainers believed that they were providing feedback to a live agent or critiquing a completed performance, and found little difference between the ways humans provided feedback in those scenarios. They also found that human trainers can decrease the consistency of feedback they give over time, however, having the agent make mistakes encouraged the human trainer to provide more feedback.

The work that bears the greatest similarity to our own is by Loftin et.al. (Loftin et al. 2014) and attempts to allow for learning of desired behavior even when the human trainer does not provide explicit feedback by using Bayesian techniques to weight silence as positive or negative depending upon the human trainers' teaching strategy. They presented two algorithms: SABL, which is explicitly told the parameters governing the trainer's teaching strategy, and ISABl, which infers the parameters of the human trainer's teaching strategy from their feedback history using maximum likelihood estimation via the Expectation Maximization algorithm (Dempster, Laird, and Rubin 1977). Significant room remains for the development of algorithms that account for these human factors while also maintaining the more precise communication abilities of a numerical reward signal. The SABL algorithms limit themselves to discrete feedback. We present a technique that handles the numerical bias that occurs in numerical feedback.

It is worth noting that there are other methods for humans to teach agents beyond giving feedback which include providing demonstrations such as in the work of Cakmak and Lopes (Cakmak and Lopes 2012) or providing a curriculum as in the work by Khan, Zhu, and Mutlu (Khan, Zhu, and
Mutlu 2011). These methods are affected by human factors in different ways and will need different techniques to address them. However, they can be combined with teaching from human feedback, and in that case our technique can be used.

\section{Methods}

Our work primarily concerns reinforcement learning. Reinforcement learning is a technique that is used to solve a Markov decision process (MDP). A MDP is a tuple $M=$ $\langle S, A, T, R, \gamma\rangle$ where $S$ is the set of world states, $A$ is the set of agent actions, $T$ is a transition function $T: S \times A \rightarrow$ $P(S), R$ is a reward function $R: S \times A \rightarrow \mathbb{R}$, and $\gamma$ is a discount factor $0 \leq \gamma \leq 1$.

The result of reinforcement learning is a policy $\pi: S \rightarrow$ $A$, which defines which actions should be taken in each state in order to maximize expected future reward.

The reinforcement learning problems we consider have the addition of a human provided feedback signal. This feedback signal is typically not included in the cumulative reward total used to evaluate an agent's performance, but may assist the agent in learning. Our technique treats the human feedback signal as being comprised of the following: 1) a heuristic assessment of the utility provided by taking an action that is a measure of both the actions immediate benefit and it's potential for long-term benefit and 2) a biasing factor that may depend upon the human trainer and aspects of the agent's presentation to the human trainer as well as the problem being learned.

Here, we assume the value of the biasing factor attached to any given feedback instance is drawn probabilistically from a distribution determined by unknown parameters. This distribution may, in some cases, be influenced by the current state or be state independent depending on the problem. Our goal is to learn the underlying heuristic evaluations present in the feedback to obtain better performance on the problems where a human trainer's feedback signal is provided but where it is highly biased. To that end, we introduce our technique, which we refer to as Bias Adaptive Statistical Inference Learning (BASIL). For the remainder of this paper, we refer to algorithms and agents that employ this technique as BASIL algorithms and BASIL agents.

At a high level, algorithms using the BASIL technique will go through a process where they use the estimation maximization algorithm by Dempster, Laird, Rubin (Dempster, Laird, and Rubin 1977) to compute a maximum likelihood estimate of the human trainer's heuristic utility evaluations using the feedback history collected by assuming as we do that feedback instances can be separated into perceived utility and bias. At a high level this consists of making an initial guess for the human's heuristic utility evaluations, from that initial guess calculating the maximum likely values for the parameters of the bias distribution, and then, from the new bias distribution parameters, calculating a new guess for the human trainer's heuristic utility evaluation. This EM step repeats until values converge within acceptable tolerances for your application. Finally, the computed estimate of the human's utility function can be used either to directly determine the agent's policy, such as in the case of the algorithm 
we present later where the technique is added to the existing TAMER algorithm, or be combined with other data, such as an environmental reward, to determine policy. Steps two through four are repeated until learning is terminated.

For the purpose of our experiments, we used the BASIL technique assuming a normal distribution for bias to develop a version of the TAMER algorithm that uses the BASIL technique to estimate an average bias in the received feedback and corrects for this bias when incorporating the feedback.

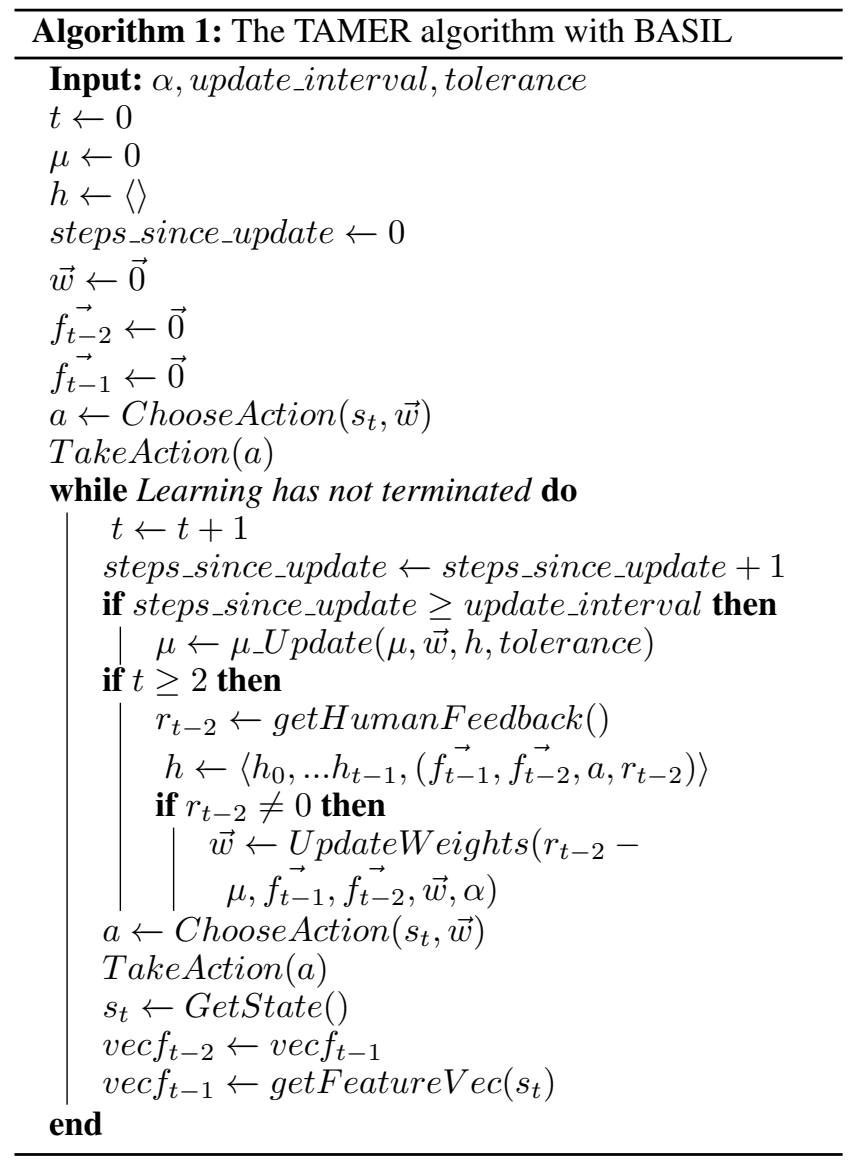

\section{BASIL TAMER Algorithm}

The classical TAMER algorithm uses a vector of weight values that correspond to the feature set chosen for a given problem. This weight vector when multiplied with the vector of features for a particular state in the problem is intended to give the value of the state as estimated by TAMER's human trainer. To learn the values of the weights, TAMER first initializes its weights to all zeros and then enters a loop where it first selects an action, according to its current weight vector, and then performs this action. If the human trainer then gives it feedback, it updates its weights by calculating the utility estimate for both the state preceding the action and the state resulting from it, taking the difference of these and comparing this difference to the feedback given by the human trainer. Assuming that these two values are not the same, TAMER then updates each weight by multiplying together

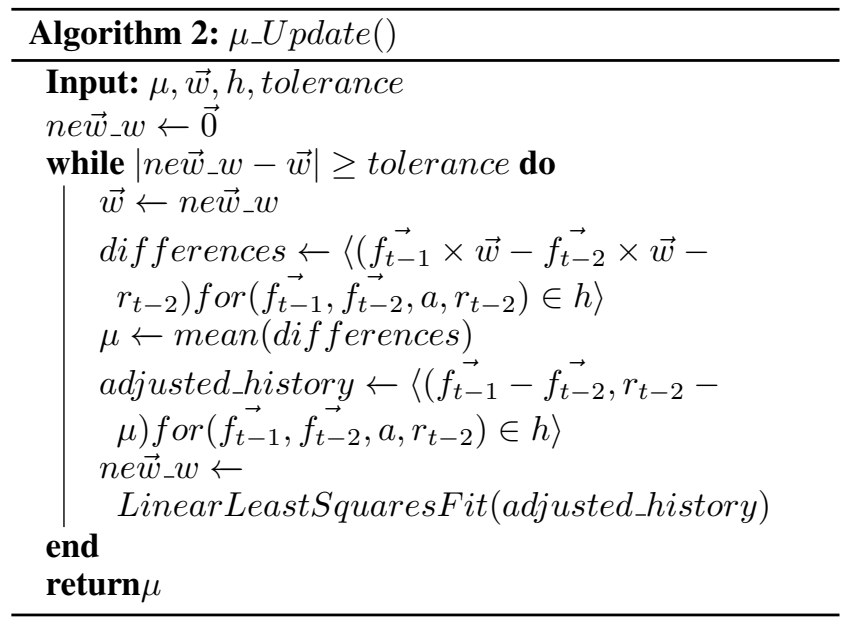

the error, the difference in feature values between the two states that correspond to the weight for each weight and a learning rate hyper-parameter, and adding the result of this multiplication to the weight whose associated feature produced it. After updating its weights, TAMER simply continues the loop until training is terminated. TAMER performs action selection by using its current weights to predict the difference in utility between its current state and the state resulting from each action and choosing the action that results in the greatest positive or least negative difference. TAMER does assume the ability to accurately predict the feature vectors of states resulting from taking each action available to it at all times.

For our BASIL TAMER algorithm with a normal distribution we make several additions. First, we address the parameters of a normal distribution. Mathematically, a normal distribution is parameterized on its median $\mu$ and variance $\sigma$ , however, variance, in this case, corresponds solely to noise in the feedback signal and, as such, cannot be helpfully addressed by BASIL techniques, as the only counter to noise is more data and a slower learning rate. Therefore, for simplicity and efficiency, this BASIL TAMER algorithm is concerned only with the median of the bias. It should be noted that this does not necessarily hold for other non-normal distributions with more complicated variance parameters. The median is represented by a variable $\mu$ initially set to zero. The TAMER algorithm is then allowed to perform its main loop for several iterations with only two changes from the classical TAMER algorithm. When receiving feedback from the human trainer the value of the feedback is adjusted by subtraction of $\mu$ before being used to update the weight vector, and the history of unmodified feedback values, feature vectors, and actions taken is stored for future use. However, after a number of iterations determined by its update interval hyper-parameter, the value of the median is recalculated using Expectation Maximization and, simultaneously, the weight vector is updated by the same process. To do this Expectation Maximization step, which is the $\mu_{-} U p$ date() step in the pseudo-code, a new weight vector is first set to zero, then a loop entered. In this loop the new weights are used 
to calculate the predicted feedback for each action the agent has taken so far in its learning history, in the same manner that TAMER normally predicts rewards. Then these predictions are compared to the actual feedback for each action. The average difference between predictions and the corresponding historical rewards is then set as the new bias median. A new weight vector is then calculated by using the newly calculated bias median to produce a modified version of the history of actions and feedbacks where the feedback values are modified by having the median bias subtracted from them. This modified history is then used to create the new weight vector. Because TAMER uses a linear model for the human trainer's utility, the new weight vector can be calculated by performing a least squared error fit for the features to the modified feedback. This process of using the weight vector to calculate the median bias and then using the median bias to calculate a new weight vector is then repeated until it converges such that the difference between the weight vectors calculated in consecutive steps is within the predetermined tolerance of the implementation.

The $\mu_{-} U p d a t e()$ step and the adjustment of the feedback received before each weight update are the only parts of the algorithm that depends upon the bias values being normally distributed. By modifying these, the algorithm can be adapted to other types of distributions.

\section{Experiments \\ Hypothesis and Metrics of Evaluation}

The goal of our experiments was to determine if adding normally distributed bias to the feedback signal given to the classical TAMER and BASIL TAMER algorithms would result in the classical TAMER algorithm failing to learn the desired behavior encoded in the feedback signal while not preventing the BASIL TAMER algorithm from learning the desired behavior. To determine the performance of the algorithms, the cumulative reward of each algorithm in an episode as a function of the number of learning episodes previously experienced was used. Such cumulative reward is a standard metric for these kinds of evaluations in reinforcement learning. In the Tetris environment, one point of environmental reward is given for each line cleared and, thus, cumulative reward for each episode is equivalent to the total number of lines cleared in that episode.

\section{Task and Environment}

The task chosen to test our BASIL TAMER algorithm was a version of the game "Tetris", modified to be suitable for reinforcement learning via conversion into a Markov Decision process. This task was chosen because it is the same task utilized in the original presentation of TAMER. (Knox and Stone 2009) In brief, Tetris is a game where the player is presented with a series of tetrominos, which are connected shapes of four blocks. The player's job is to rotate and move the tetrominos until they contact a placed tetromino or the bottom of the play field, at which point they lock in place. When a row is completely filled, the blocks in that row disappear and blocks in all higher rows fall by one level. While various renditions of the game use different point scoring schemes, in general, the goal is to clear as many rows as possible. In reinforcement learning friendly formulations of the game, the player's options for actions to take consist of each possible valid placement of the currently active tetromino and the player has no knowledge of upcoming tetrominos so that the problem is an MDP. For our experiment, both classic TAMER and BASIL TAMER, utilize a vector of features that describe the current game state and are pre-calculated for them. These features are: the heights of each column; the height differences between each pair of adjacent columns; the height of the highest column; the total number of holes in the play field, that is empty squares with full squares above them; and a fixed weight for computational purposes. This feature set has been used successfully in many works including the original presentation of TAMER. (Knox and Stone 2009)

\section{Protocols}

An evaluation episode was preformed after each training episode to test the current quality of the learned policy. Each agent received 20 training episodes. In the bias free case it takes under 6 episodes for both algorithms to match the oracle's performance, and this result is similar to what was observed in Knox and Stone's original publication of the TAMER algorithm. (Knox and Stone 2009) During evaluation episodes, learning does not occur and all actions are taken according to the agents' policies. Both training and evaluation episodes were limited to 3000 steps. To counteract the non-determinism inherent in Tetris, each algorithm was instantiated with 30 independent agents that were trained separately, and the average of the cumulative rewards for each agent for each evaluation episode was taken to obtain the score for each algorithm as a function of the number of training episodes undergone.

\section{Simulated Human Trainers}

We use simulated human trainers in our experiments. We choose to use simulated human trainers because this allows us to both know and set the ground-truth for the feedback and bias provided in our experiments and makes our experiments significantly more reliable and reproducible then they could be using live humans. This is a common practice in interactive reinforcement learning work. The simulated human trainers used in our experiments provided feedback in two parts that were combined before being given to the agents. The first part is the utility of the action taken as evaluated by a heuristic function. The second part is a bias value drawn from a normal distribution. The utility of taking an action for a particular state is determined by the weighted sum of the difference of feature vectors for the state that preceded the action and the resulting state. The second part is the bias which is drawn from a normal distribution and is defined by the mean of the normal distribution of the bias and the variance of the normal distribution.

\section{Preliminary Experiments}

We conducted preliminary experiments comparing a BASIL Q-Learner, TAMER, and ISABL in a grid world environment. We do not have the space to discuss them in this work, 


\begin{tabular}{l|l|l|l}
$\mu$ & $\sigma$ & Classic TAMER & BASIL TAMER \\
0.0 & 0.0 & 75.2 & 74.3 \\
5.0 & 0.0 & 0.0 & 73.5 \\
20.0 & 0.0 & 0.0 & 73.8 \\
80.0 & 0.0 & 0.0 & 71.7 \\
-5.0 & 0.0 & 49.0 & 73.8 \\
-20.0 & 0.0 & 3.5 & 76.2 \\
-80.0 & 0.0 & 1.5 & 75.6 \\
0.0 & 3.0 & 15.2 & 14.1 \\
5.0 & 3.0 & 0.0 & 16.5 \\
20.0 & 3.0 & 0.0 & 14.7 \\
80.0 & 3.0 & 0.0 & 14.0 \\
-5.0 & 3.0 & 36.8 & 17.0 \\
-20.0 & 3.0 & 4.4 & 17.6 \\
-80.0 & 3.0 & 1.3 & 18.4 \\
0.0 & 30.0 & 1.3 & 0.4 \\
5.0 & 30.0 & 1.1 & 0.7 \\
20.0 & 30.0 & 0.1 & 0.7 \\
80.0 & 30.0 & 0.0 & 1.1 \\
-5.0 & 30.0 & 1.4 & 1.5 \\
-20.0 & 30.0 & 1.3 & 1.0 \\
-80.0 & 30.0 & 2.1 & 0.4
\end{tabular}

Table 1: Averages over the last ten episodes of training across all samples for each TAMER algorithm for each permutation of bias parameters.

but wish to explain why we do not use ISABL as a baseline here. In brief these preliminary experiments demonstrated that ISABL is entirely unsuited for the type of bias we are addressing.

\section{Results and Analysis}

As mentioned previously, we tested on 21 permutations of the parameters controlling the simulated human trainer's feedback and due to space constraints, we will have to limit our analysis here to illustrative examples. Averages of the performance of each algorithm under each permutation of basis for the last 10 episodes of training are provided in Table 1. Our full results for all experiments are available in supplemental files.

\section{Perfectly Reliable Feedback}

As can be seen in Figure 1, when the simulated human trainers give perfectly reliable feedback with no bias both TAMER and BASIL TAMER algorithms preform very well and match the oracle weights performance before the 5 th episode.

\section{Normally Biased Feedback}

Adding a moderate positive bias such as 5.0 causes classic TAMER to completely fail as can be seen in Figure 2. However, it is worth mentioning that for this problem classic TAMER did better with negative bias and as can be seen in Table 1 maintained a decent performance at -5.0 median bias value although its performance was still negatively impacted with more extreme negative bias having

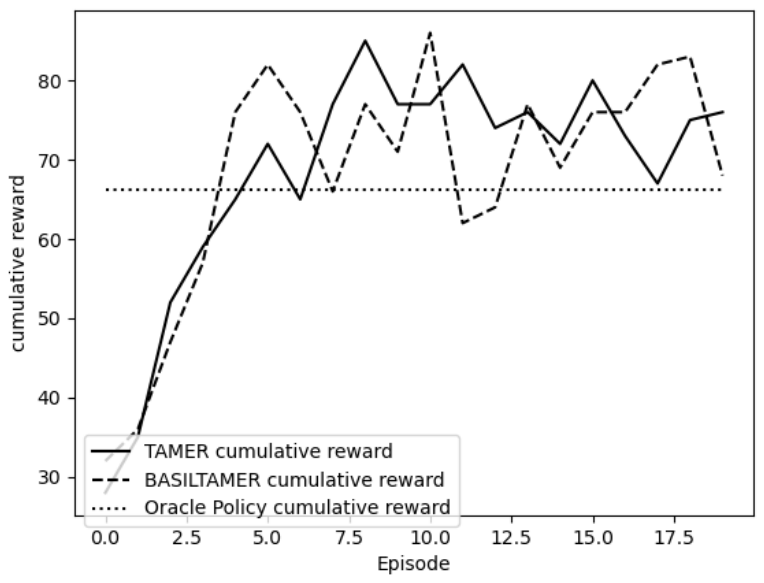

Figure 1: Cumulative Reward for each algorithm when feedback contains no bias.

greater consequences. We believe the difference in performance for negative verse positive bias can be attributed to the feature set which is such that all features in it should generally be minimized as opposed to maximized and therefore feedback corresponding high performance such as that given by the oracle will be almost entirely negative. BASIL TAMER maintains its performance at all bias values even with very high bias values such as 80.0 regardless of the direction of bias.

\section{High Variance in Feedback}

As can be seen in Table 1, having higher than expected variance in the feedback signal is a problem for both forms of TAMER we tested. This is to be expected as this variance amounts to noise in the feedback signal and the only counter to this is to use a smaller alpha value and collect more feedback instances. Supplemental experiments were performed with the $\mu=0.0$ and $\sigma=3.0$ values using smaller values for alpha and this was confirmed to restore eventual performance to the levels of the $\mu=0.0$ and $\sigma=0.0$ case. Countering variance dynamically is a problem for future work.

\section{Discussion}

As demonstrated in the results, the BASIL technique allows for very rapid learning under even very heavy biases that warp all human feedback to the negative or positive reward values. This is not without cost. It is necessary to choose a correct distribution type for the bias modeling. Depending upon the distribution chosen and the way bias is actually distorted, you may get better results accounting for the wrong type of bias than for not accounting for bias at all; however, this is not guaranteed. The BASIL technique is only applicable for distribution types where the maximum likelihood function can be calculated. For some problems, performing the EM calculation may be costly. This may be offset by not preforming it at every new instance of feedback. It is up to 


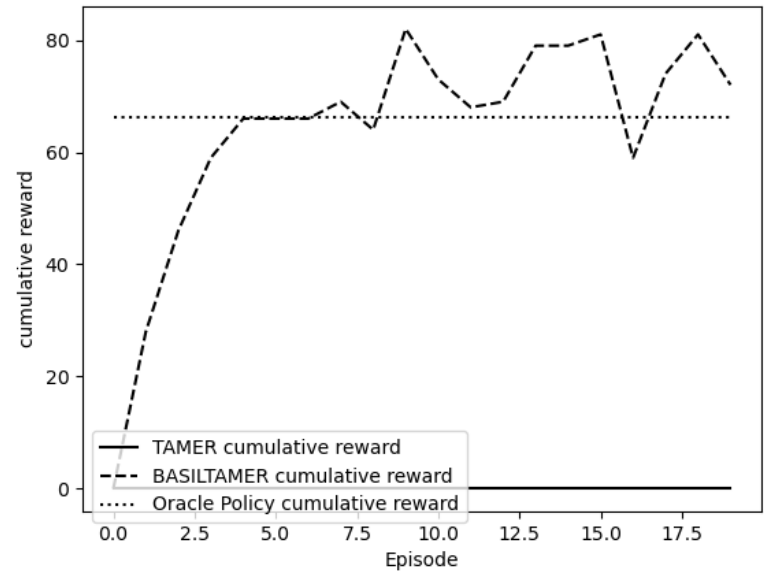

Figure 2: Cumulative Reward for each algorithm when feedback contains moderately high positive bias $\mu=5.0$ with no variance $\sigma=0.0$.

an agent's engineers to find a balance that works for their problem. In our experiments, we tested only one algorithm using the BASIL technique. There are many ways in which the technique could be used in other algorithms. For example, there is no reason an engineer could not develop an algorithm that uses feedback provided by a human trainer filtered with the BASIL technique in addition to environmental reward.

\section{Future Work}

There is significant room for further development of algorithms that use the BASIL technique. Our testing only covered normally distributed bias however, the BASIL technique is theoretically applicable for any bias for which the maximum likelihood function is computable.

Another area for future work is experiments using feedback provided by actual humans. Determining the nature of the bias in feedback provided by humans is challenging because the ground-truth of their heuristic assessment of the utility of actions is unknown. Using BASIL based algorithms designed for different distribution forms, experiments could be performed into what bias distribution model produces the best results in various scenarios.

As mentioned in the Discussion, the BASIL technique can theoretically be adapted to work with multiple bodies of feedback with different bias distribution parameters, or, theoretically, even entirely different forms of bias distribution as long as it is reasonable to model the feedback as being based on the same estimates of state action pair utility. The development and testing of concrete algorithms using this variant of the BASIL technique is an area for future work.

\section{Conclusion}

Interactive Reinforcement Learning is a variant of reinforcement learning that includes humans as an additional source of information to learn behaviors from. On many tasks, it can allow agents to learn effective behavior much more quickly than using classical reinforcement learning. However, introducing humans directly into the process introduces complicated human factors that can skew the feedback given and may vary depending upon the humans in questions, the presentation of the agent and the problem to them, and myriad other factors. We presented a general technique called Bias Adaptive Statistical Inference Learning (BASIL) to filter out bias from feedback. We presented an algorithm using the BASIL technique applied to the existing TAMER algorithm for normally distributed bias and tested it against classic TAMER in the domain of Tetris. We found that our algorithm, using the BASIL technique, was able to filter both positive and negative bias out of feedback provided by simulated human trainers. This allowed our algorithm to perform well on highly distorted feedback that rendered classic TAMER incapable of learning how to clear any lines. We discussed ways to integrate the BASIL technique into other interactive reinforcement learning work and the options it provides for studying the bias in human feedback in the future.

\section{References}

Bartneck, C.; Reichenbach, J.; and Carpenter, J. 2008. The carrot and the stick: The role of praise and punishment in human-robot interaction. Interaction Studies 9(2):179-203. Cakmak, M., and Lopes, M. 2012. Algorithmic and human teaching of sequential decision tasks. In AAAI.

Dempster, A. P.; Laird, N. M.; and Rubin, D. B. 1977. Maximum likelihood from incomplete data via the em algorithm. In Journal of the Royal Statistical Society. Series B (Methodological), volume 39, 1-38.

Khan, F.; Zhu, X.; and Mutlu, B. 2011. How do humans teach: On curriculum learning and teaching dimension. In NIPS.

Knox, W. B., and Stone, P. 2009. Interactively shaping agents via human reinforcement: the tamer framework. In $K$ - $C A P$.

Knox, W. B.; Glass, B. D.; Love, B. C.; Maddox, W. T.; and Stone, P. 2012. How humans teach agents - a new experimental perspective. International Journal of Social Robotics 4:409-421.

Loftin, R. T.; MacGlashan, J.; Peng, B.; Taylor, M. E.; Littman, M. L.; Huang, J.; and Roberts, D. L. 2014. A strategy-aware technique for learning behaviors from discrete human feedback. In AAAI, 937-943.

Sutton, R. S.; Barto, A. G.; et al. 1998. Introduction to reinforcement learning, volume 2. MIT press Cambridge.

Thomaz, A. L., and Breazeal, C. 2006. Transparency and socially guided machine learning. In 5th Intl. Conf. on Development and Learning (ICDL). 